\title{
Accuracy and Completeness of Medication Histories in Patients in Medical Admission Ward at University Teaching Hospital
}

\author{
Mutinta M1, Muungo L.T1, Yassa P2 \\ 1The University of Zambia, School of Medicine, Department of Pharmacy, Lusaka, Zambia. \\ 2Cavendish University, School of Medicine, Lusaka, Zambia. \\ Corresponding Author: \\ University of Zambia, School of Medicine, Department of Pharmacy, P.O. Box 50110, Lusaka, Zambia. \\ Mobile number: 0966743914/0955786226 Email: melodymbewe@yahoo.com
}

\begin{abstract}
Background:Qualitydocumentation ofmedication histories at the time of hospital admission with regard to accuracy and completeness is not documented at the University Teaching Hospital (UTH), in Zambia. Our study aimed to assess the accuracy and completeness of medication histories obtained in patients upon hospital admission.
\end{abstract}

Materials and Methods: We conducted a prospective cross-sectional study atmedical admission ward, University Teaching Hospital, over a period of 3 months. Our study enrolled 322 patients admitted to this ward who were above 18 years of age and could communicate verbally, if not, were accompanied by a caregiver. These patients' clinical records were screened to review all medications the patient was taking, and patients/ caregivers were interviewed to obtain a complete medication history. All information obtained from patients through interviews was compared with medications recorded in the patient's clinical records when admitted to the hospital. The Statistical Package for Social Sciences(SPSS) version 22 was used for all statistical calculations.

Results: Of 287 clinical records, 175 (61\%) incidents of inaccurate medication histories at the time of admission were identified, and that medication histories in clinical records of patients were incomplete or poorly documented.

Conclusion: Our study shows that $61 \%$ of medication histories in patients at the time of admission to hospitals are inaccurate. Quality documentation of medication histories in clinical records at the time of hospital admission is poor.

Keywords: Accuracy of medication history, complete medication history, medication history, Medication, Medication discrepancy

\section{INTRODUCTION}

Medication-related hospital admissions account for $2-4 \%$ of all admissions in Australia, with higher rates (above 30\%) in the elderly above 75 years, for unintended admissions, most of which are preventable. ${ }^{1} \mathrm{~A}$ medication history is a detailed, accurate and complete account of all currently and recently prescribed and nonprescribed medications that a patient had taken before a newly established or ambulatory care. Globally, inaccurate and incomplete medication histories at hospital admission do exist with a high rate of errors ${ }^{2}$ which can considerably harm patients. ${ }^{3}$ Studies done in North America, Europe, Australia and Asia to describe the extent of inaccurate medication histories at the time of hospital admission showed that up to $67 \%$ of patients under study have at least one medication history error. ${ }^{2,45,6,7,8,9}$, Abu -Yassin and Colleagues reported a relatively scanty published literature on this subject in Africa. ${ }^{9}$

Barnsteiner foresaw the need for a study on all parts of the medication reconciliation process to provide an evidence base for addressing adverse drug events. ${ }^{10}$ A recent study showed that in Saudi Arabia, inaccurate medication histories at the time of hospital admission were common; however, the results might be considerably different in other developing countries as studies of this nature are lacking. ${ }^{9}$ Cornish et al. believed that better methods to ensure accurate admission medication histories were needed to improve patient care and minimise adverse drug events. Greenwald et al. ${ }^{11}$ supported the need for studies to assess the potential solutions to overcome these and other common barriers. ${ }^{12}$

A study done at a tertiary care teaching hospital by Cornish and Colleagues in Canada in 2003 on patients admitted to the general internal medicine units with 151 eligible patients showed that that $54 \%$ had medication history errors. From these, 
$39 \%$ had the potential to cause moderate to severe discomfort or clinical deterioration. ${ }^{11}$ Unroe et al. upon examining 205 patient records of a tertiary care academic teaching hospital in Durham, North Carolina in 2005 found that while 178 patients had medications listed, $23 \%$ of these had one or more discrepancy identified on admission; 19\% of these were considered to be potentially harmful. ${ }^{8}$ In a study carried out in 2009 at a 1200 bed tertiary hospital in Riyadh, Saudi Arabia, by Abu-Yassin et al., 37\% of patients were found to have at least one discrepancy in their admission medication histories, with the most common being omissions of medications (35\%) and dosage errors $(35 \%) .{ }^{9}$ in Nigeria, a low level of medication history documentation was reported in patients before admission. $^{13}$

Our study intended to assess the accuracy and completeness of medication histories obtained in patients upon hospital admission. An accurate and comprehensive medication history taking the approach that included an interview, inspection of medication containers or lists, or both, documenting the patient's medication history was used, and this was compared with medications recorded in the patient's clinical records(i.e. medical notes and drug chart) at the time of admission to the hospital.

\section{MATERIALS AND METHODS}

Our study was a cross-sectional study conducted at the University Teaching Hospital, Medical Admission Ward, Lusaka, from January 2015 to March 2015. A total of 322 participants meeting the eligibility criteria of 18 years of age and above, able to communicate verbally, if not, were accompanied by a caregiver were included. Participants, less than 18 years of age, unable to consent, outpatients and those in isolation rooms were excluded from the study. ${ }^{11}$

Patients were identified from the Medical admission register. If eligible, interviews were conducted, including examining medication vials (if available) to obtain a complete medication history. The interviews were conducted generally on the day after the admission at the bedside of the patient. Patient's clinical records were screened to review all medications the patient was on before hospital admission. Data obtained from interviews was compared with that on the patient's clinicalrecords. ${ }^{5,9}$
A Statistical Package for Social Science (SPSS) software, version 22 (SPSS Inc., Chicago, IL, USA) was used to analyse data. For open-ended questions and clinical notes reviews, data was captured, analysed qualitatively and then entered quantitatively into SPSS. Data were expressed as frequency and percentage and presented using tables. A cross-tabulation of Accuracy of medication histories and Completeness of documentation was executed using the Pearson chi-square test, $p<0.05$ was considered statistically significant.

Fully informed written consent was sought from all participants following thestudy'sclearancefrom ERES CONVERGE IRB Ethics Committee (Ref. No. 2014-Sept-009) and the University Teaching Hospital management.

\section{Definitions}

Our definition of an accurate medication history was based on the definition by Gleason et al. ${ }^{14}$ " $\mathrm{A}$ complete matching of the medication name, dose, route and frequency of the two lists, i.e. one obtained by the admitting physician and that obtained after admission through interviews and other sources by another clinician, e.g. pharmacist."

Complete medication history encompassed all currently and recently prescribed medications (including vaccines, diagnostic and contrast agents, radioactive medications, parenteral nutrition, blood derivatives, and intravenous solutions), samples from your doctor, and any medications bought without a prescription, including over-thecounter medications (OTC), vitamins, and herbal supplements. ${ }^{14,15}$

We used the Joint Commission 2010's definition of medication as "any prescription medications, sample medications, herbal remedies, vitamins, nutraceuticals, vaccines, or OTC; diagnostic and contrast agents used on or administered to persons to diagnose, treat, or prevent disease or other abnormal conditions; radioactive medications, respiratory therapy treatments, parenteral nutrition, blood derivatives, and intravenous solutions (plain, with electrolytes or drugs); and any product designated by the Food and Drug Administration (FDA) as a drug." 16

We adopted Collins et $a l^{17}$ definition of medication discrepancy. "Any aspect of medication prescribing not recorded by the admitting physician but is recorded in the pharmacy researcheracquired medication history." 


\section{RESULTS}

\section{Characteristics of the study participants}

Of the 322 patients that met the eligibility criteria, $154(47.8 \%)$ were male, $27(8.4 \%)$ were not on any medications before hospital admission, and $25(7.8 \%)$ patients did not have any medication histories in their clinical notes hence, the accuracy of medication history was not determined in such. We interviewed 171 (53.1\%) patients and 151 $(46.9 \%)$ caregivers.

\section{Determination of accuracy of medication histories at the time of hospital admission}

Of the 287 medication histories, 112 (39.1\%) were accurate as no discrepancies were noted in medication name, dose, route and frequency of administration (Table 1).

Table 2 shows the number of discrepancies (inaccuracies) that were identified during the review of clinical records. The most common discrepancy was medication omissions at $27.2 \%$, while dose omission, route, and administration frequency at $11.8 \%, 18.5 \%$ and $11.8 \%$ respectively. More than one discrepancy per medication history was reported in some instances; therefore, they were grouped under others $(8.4 \%)$. In some cases, patients were issued with prescriptions without the medications being captured in their clinical records (10.8\%). Some medications were documented on drug charts and not in medical notes $(4.9 \%)$ and vice-versa $(2.1 \%)$.

Determination of completeness of documentation of medication histories in clinical records at the time of admission

The study showed that medication histories in patients' clinical records in the medical admission ward were poorly documented, as shown in Table 3. POM, OTC, and CAM accounted for $55 \%$, $12.4 \%$ and $7.5 \%$ respectively, whereas, Social history, ADRs and allergies were documented in $12.7 \%, 2.2 \%$ and $1.9 \%$ respectively. Adherence and other groups (such as samples, vaccines, and ENT) were recorded in $0.3 \%$ and $0.6 \%$. Of the 322 clinical records, $7.8 \%$ did not have medication histories indicated.

Determination of the association between accuracy and completeness of documentation of medication histories in clinical records at the time of admission.
There was an association between accuracy and completeness of documentation of medication histories in clinical records at the time of admission $(\mathrm{p}=0.001)$

\section{DISCUSSION}

Determination of accuracy of medication histories at the time of hospital admission

Our study shows that medication histories in patients at the time of hospital admission are inaccurate. Of the 287 medication histories examined, $61 \%$ were inaccurate. This was consistent with the findings of a systematic review of 22 studies undertaken by Tam et al., which reported medication history errors to vary between 10 and $67 \% .^{2}$ The study carried out by Miller and Colleagues found a much higher percentage $(96 \%)$ probably due to a longer medication reconciliation period (1-8 days) that was involved, ${ }^{18}$ unlike most studies (within 72 hours of admission). ${ }^{9,11}$

Our study found at least one medication history discrepancy present in $61 \%$ medication histories. The predominate discrepancy was medication omissions $(27.2 \%)$ although it was lower than what other pooled data from other studies like Cornish et al. (46\%) and Crook et al., (90\%) showed. ${ }^{7,11}$ The results of our study also have shown discrepancies in dose omission, route, and frequency of administration at $11.8 \%, 18.5 \%$ and $11.8 \%$ respectively

During the interviews, some of the patients' medications were still taking were identified as we also inspected medication containers. It was noticed that these medications were not captured anywhere in clinical records $(10.8 \%)$. In some cases, patients were issued with prescriptions without the medications being captured in their clinical records.

The outcome of the review of the clinical records further demonstrated that admitting physicians documented medications on drug charts and not in medical notes $(4.9 \%)$ and vice-versa $(2.1 \%)$. This is in line with what Collins et al. found in their U.K. study. ${ }^{17}$ Medications were documented in medical notes without indicating the dosage, route and the frequency of administration (8.7\%); however, some of these were captured on the drug charts. We found physicians overlooking dose, route, and frequency of administration at $11.8 \%$, $18.5 \%$ and $11.8 \%$ respectively in clinical notes corresponding to what was obtained in a study by Miller and Colleagues. ${ }^{18}$ 
In a similar study in Slovenia, Re'onjaet al. reported more than one discrepancy per medication history similar to this study, this was difficult to analyse (therefore, were grouped under others). ${ }^{19}$ Other discrepancies (8.4\%) included wrong frequency of administration, wrong drug descriptions or names, incomplete dose, and differences between clinical notes.

Determination of completeness of documentation of medication histories in clinical records at the time of admission

This study provides evidence that medication histories in clinical records at the time of hospital admission at UTH are incomplete. There was poor documentation of POM, OTC and CAM accounting for $55 \%, 12.4 \%$ and $7.5 \%$ of completeness respectively. Social history, ADRs and allergies were documented in $12.7 \%, 2.2 \%$ and $1.9 \%$ respectively. Adherence and other groups (such as samples, vaccines, and ENT were recorded in $0.3 \%$ and $0.6 \%$. The results of our study are comparable with those of Yusuf, and Awotundealthough study designs were different. ${ }^{13}$ Equally, Re'onjaet al. reported a high level of incomplete information on drug use in the medical record. ${ }^{19} \mathrm{~A}$ study that was conducted by Unroe et al. showed that $13.2 \%$ of patients did not have their medications recorded on admission, whereas in this study $7.8 \%$ did not have medication histories in their clinical records. ${ }^{8}$ Some limitations were identified with the study. The study was conducted at a single centre (Medical admission ward); this made it difficult to generalise the findings to other hospitals. Of the 334 eligible participants, 322 gave a full response.

This study's findings showed that medication histories in clinical records of patients at the time of admission to hospitals are generally inaccurate (61\%) and incomplete. It can also be concluded that the completeness of medication history documentation affects medication history accuracy $(p=0.001)$, as shown in Table 4 .

Based on the study's findings, the authors recommend using a standardised form to be used by physicians on admission, which should capture all the requirements of complete medication history. This should be attached to the patients' file, where the information will be accessible (Table 5). Admitting physicians need to be sensitised about the importance of recording an accurate and complete medication history of the patients.

Clinical pharmacists should be engaged in documenting medication histories of patients on admission. This has been indicated by several studies that have been done in most developed countries. ${ }^{9,20,21,22}$

There is a need to carry out a study at multi centres for the results to be generalised and to know the association between the level of practice and drug history taking.

\section{Acknowledgements}

Our gratitude goes to Mr Mwangana Mubita, and Mr Jimmy Hangomafor their helpful comments that encouraged and confronted us with being innovative in our research and; UTH medical admission ward staff for their cooperation during the process of data collection.

\section{REFERENCES}

1. Runciman WB, Roughead EE, Semple SJ, Adams RJ. Adverse drug events and medication errors in Australia.Int $\mathrm{J}$ Qual Health Care2003; 15(1);i49-i59

2. $\mathrm{m}$ VC, Knowles SR, Cornish PL, Fine N, Marchesano R, Etchells EE.Frequency, type and clinical importance of medication history errors at hospital admission: a systematic review.CMAJ.2005; 173:510-5.

3. FitzGerald RJ. Medication errors: the importance of an accurate drug history. Br $\mathrm{J}$ Clin Pharmacol. 2009; 67(6): 671-675.

4. Cockayne NL, Duguid M, Shenfield G.M. Health professionals' rarely record the history of complementary and alternative medicines. Br J Clin Pharmacol. 2005; 59(2); 254-258.

5. Vira1 T, Colquhoun M, Etchells.Reconcilable differences: correcting medication errors at hospital admission and discharge.QualSaf Health Care 2006; 15;122-126

6. Crook M, Ajdukovic M, Angley C, Soulsby N, Doecke C, Stupansi I, et al.Eliciting comprehensive medication histories in the emergency department: the role of the Pharmacist. Pharmacy Practice (Internet) 2007; 5(2) Redondela abr.

7. Unroe KT, Pfeiffenberger $\mathrm{T}$, Riegelhaupt $\mathrm{S}$, Jastrzembski J, Lokhnygina Y, ColonEmeric C.Inpatient medication reconciliation at admission and discharge: a retrospective cohort study of age and other risk factors for medication discrepancies. Am J GeriatrPharmacother. 2010;8(2): 115-126. 
8. Abu-Yassin BH, Aljadhey H, Al-Sultan M, Al-Rashed S, Adam M, Bates DW. Accuracy of the medication history at admission to hospital in Saudi Arabia.Saudi Pharm J 2011; 19(4); 263-267.

9. Hellström LM, Bondesson A, Höglund P, Eriksson T. Errors in medication history at hospital admission: prevalence and predicting factors.BMC Clin Pharmacol. 2012; 12; 9.

10. Barnsteiner JH. Patient Safety and Quality: An Evidence-Based Handbook for Nurses. Chapter 38 Medication Reconciliation. Editor: Hughes, RG. Apr 2008. Available from: <http://www.ahrq.gov/professionals/ clinicians/nursing/...BarnsteinersJMR.pdf> (March 20 2014)

11. Cornish PL, Knowles SR, Marchesano R. Unintended medication discrepancies at the time of hospital admission. Arch. Intern. Med. 2005; 165(4); 424-429.

12. Greenwald JL, Halasyamani L, Greene J, LaCivita C, Stucky E, Benjamin B, et.al. Making Inpatient Medication Reconciliation patient-centred, Clinically Relevant and Implementable: A Consensus Statement on Key Principles and Necessary First Steps. J Hosp Med.2010; 5(8) 477-485.

13. Yusuff K, Awotunde M. The frequency of drug history documentation in an institutionalised tertiary care setting in Nigeria. J Pharm Pharmaceut Sc.2005; 8(2); 141-146.

14. Gleason KM, Brake H, Agramonte V, Perfetti C.Medications at Transitions and Clinical Handoffs (MATCH) Toolkit for Medication Reconciliation. Agency for Healthcare Research and Quality (AHRQ) 2012; 11(12) 0059 .
15. JCAHO. National Patient Safety Goals. 2005. Available from: <http://www .jointcommission .org/PatientSafety/ NationalPatientSafetyGoals/05_npsgs.htm> ( $9^{\text {th }}$ April 2014).

16. Accreditation program: hospitals. Oakbrook Terrace (I.L.): The Joint Commission; 2010, p. G.L. 19. National patient safety goals. Effective January 1, 2011. Available from: <http://www.jointcommission.org/ assets/1/6/2011_NPSGs_HAP.pdf. $>\left(9^{\text {th }}\right.$ April 2014)

17. Collins DJ, Nickless GD, Green C.F. Medication histories: does anyone know what medicines a patient should be taking? International Journal of Pharmacy Practice. 2004; 12(4); 173-178.

18. Miller SL, Miller S, Balon J, Helling TS. Medication reconciliation in a rural trauma population. Ann Emerg Med. 2008; 52(5); 483-491.

19. Re'onja R, Knez L, Suskovic S, Kosnik M, Mrhar A.Comprehensive medication history: the need to implement medication reconciliation processes. 2010; Original scientific article UDC 001.102:615.11:614.21

20. Carter MK, Allin DM, Scott LA, Grauer D. Pharmacist-acquired medication histories in a university hospital emergency department.Am J Health Syst Pharm.2006; 63(24); 2500-3.

21. Reeder TA, Mutnick A. Pharmacist- versus physician-obtained medication histories.Am J Health Syst Pharm. 2008; 65(9); 857-60

22. De Winter $S$, Spriet I, Indevuyst $C$, Vanbrabant P, Desruelles D, Sabbe M, et al. Pharmacist- versus physician-acquired medication history: a prospective study the emergency department.QualSaf Health Care. 2010;19:371-375 


\section{ANNEX}

Table 1: Accuracy of medication histories at the time of hospital admission.

\begin{tabular}{|l|c|c|}
\hline & Frequency & Percent \\
\hline Accuracy & 112 & 39.07 \\
\hline Inaccuracy & 175 & 60.97 \\
\hline Total & 287 & 100 \\
\hline
\end{tabular}

Table 2: Types of discrepancies

\begin{tabular}{|c|c|c|}
\hline Type of discrepancy & Frequency & Percent \\
\hline Medication Omissions & 78 & 27.2 \\
\hline Dosage omission & 34 & 11.8 \\
\hline Route omission & 53 & 18.5 \\
\hline Frequency omission & 34 & 11.8 \\
\hline Meds* on chart but not doc* in notes $^{*}$ & 14 & 4.9 \\
\hline Meds $^{*}$ in notes but not doc ${ }^{* *}$ on chart & 6 & 2.1 \\
\hline Meds* identified from interviews but not in & 31 & 10.8 \\
\hline \multicolumn{3}{|l|}{ Clinical records } \\
\hline Wrong dose & 8 & 2.8 \\
\hline 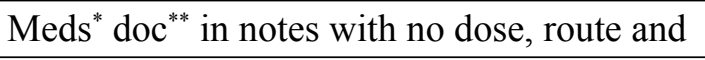 & 25 & 8.7 \\
\hline Others & 24 & 8.4 \\
\hline
\end{tabular}

Table 3: Completeness of documentation of medication histories

\begin{tabular}{|l|c|c|}
\hline Prescription-only medications & 177 & 55 \\
\hline Over-the-counter & 40 & 12.4 \\
\hline Complementary and alternative medicines & 24 & 7.5 \\
\hline Social history (alcohol, smoking, illicit drugs) & 41 & 12.7 \\
\hline Adverse drug reactions & 7 & 2.2 \\
\hline Allergies (food, drugs, chemicals) & 6 & 1.9 \\
\hline Adherence & 1 & 0.3 \\
\hline Others & 2 & 0.6 \\
\hline & & \\
\hline
\end{tabular}

Table 4: Association between the accuracy of medication histories and completeness of documentation

\begin{tabular}{|l|l|l|l|}
\hline & Value & df & Standard deviation \\
\hline Pearson chi-square & $140.322^{\text {a }}$ & $\mathbf{6}$ & 0.001 \\
\hline Likelihood ratio & 89.199 & $\mathbf{6}$ & 0.000 \\
\hline N of Valid cases & 322 & & \\
\hline
\end{tabular}


Table 5: Proposed Medication History Documentation Form

MINISTRY OF HEALTH

\section{University Teaching Hospital, Private Bag R.W. 1X, Lusaka}

This form must be completed by practitioners clerking in patients on admission and filed in the patients file. Additional information can be added as it becomes available.

\section{Patients' details}

Name:

Date of birth

File No:

Source of information: (Tick)

Patient ( ) / Caregiver ( )

Recent discharge ( ) date.

Other ( ) specify.
Allergies: (Tick and include reaction details)

Medication ( )

Chemicals ( )

Food ( ).

Medications on admission (including herbal, vitamins and over-the-counter)

\begin{tabular}{|l|l|l|l|l|}
\hline Medication name & Dose & Route & Frequency & $\begin{array}{c}\text { Comments, e.g. reasons why any } \\
\text { medication is not prescribed }\end{array}$ \\
\hline & & & & \\
\hline & & & & \\
\hline & & & & \\
\hline & & & & \\
\hline & & & & \\
\hline
\end{tabular}

\section{Additional information needed:}

\begin{tabular}{|l|l}
\hline Social history: (Tick) & Adherence: (Tick) \\
Alcohol ( ) & Poor compliance ( ) \\
Smoking ( ) & Medications recently stopped/courses completed ( ) \\
Illicit drugs ( ) & Patient brought medicines to the hospital ( )
\end{tabular}

\begin{tabular}{|l|l|l|l|l|l|}
\hline & Print name & Designation & Signature & Date & Time \\
\hline Completed by & & & & & \\
\hline $\begin{array}{l}\text { Amendments } \\
\text { made by }\end{array}$ & & & & & \\
\hline
\end{tabular}

